\title{
El éxito evolutivo de los recolectores de caracoles de la Puebla del Río
}

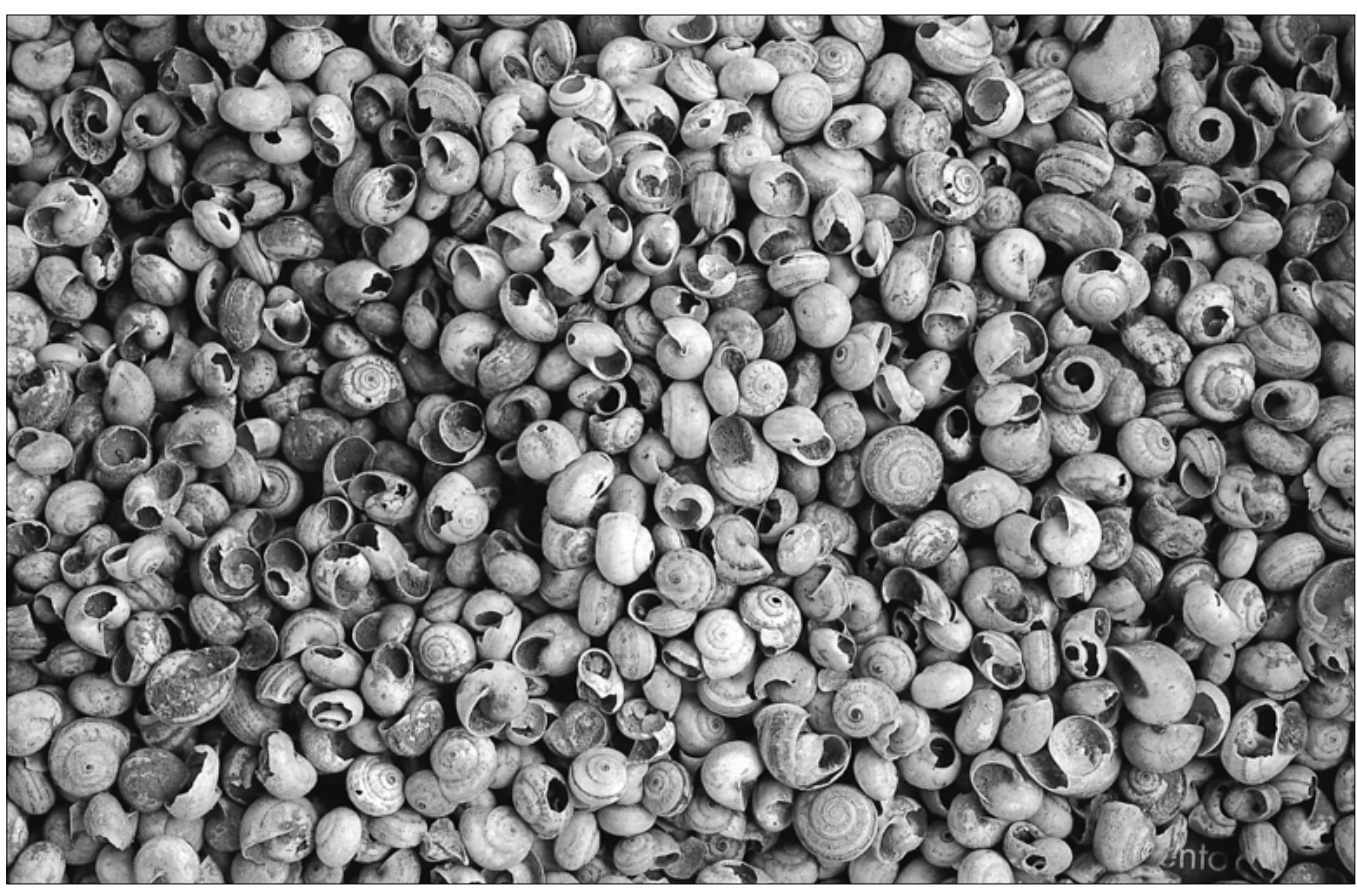

Caracoles hallados en el fondo de cabaña datado en el siglo VII a.C. del yacimiento arqueológico situado en el Cerro de la Albina en la Puebla del Río -SevillaFoto: Eugenio Fernández

\section{Eloísa Bernáldez Sánchez \\ María Bernáldez Sánchez}

Laboratorio de Paleobiología del IAPH

\section{Palabras clave}

Tafonomía / Bioestratinomía / malacofauna / tanatocenosis

\section{Resumen}

La recolección de caracoles para el consumo es una actividad actual cuya eficacia fue alcanzada, al menos, por quienes recolectaron los más de 6000 caracoles conservados en el fondo de una cabaña del siglo VII a.C. situada en la localidad de La Puebla del Río (SeviIla). El estudio tafonómico y bioestratinómico nos ha permitido precisar las características de la recolección y los posibles cambios ambientales que justifican las condiciones tafonómicas del hallazgo: bolsadas con similar número de caracoles correspondientes a sólo tres especies, muchos de ellos agujereados.

\section{¿Por qué seguimos comiendo caracoles en Andalucía?}

¿Por qué en Andalucía seguimos comiendo caracoles cuando podemos comer otros alimentos por el mismo precio? Que los comiesen en el siglo VII a.C. en La Puebla del Río, como así parece que hicieron en vista del hallazgo en escasos $\mathrm{m}^{3}$ de más de $6.000 \mathrm{ca-}$ racoles adultos, puede que fuese un acto de supervivencia; pero lo que es indiscutible es que hoy en día los andaluces no comen caracoles por carencia de 
otras fuentes de proteína animal. En Sevilla un Kg de caracolillos -Helix aspersa- vale unas 400 ptas y de cabrillas -Otala lactea- unas 500 ptas, por estos precios se puede comprar un cuarto de solomillo de ternera de primera calidad o un $\mathrm{Kg}$ de carne de vacuno sin preparar; un $\mathrm{Kg}$ de costilla de cerdo o de falda o tres cuartos $\mathrm{Kg}$ de cordero.

No sabemos cuánto de básico tiene el consumo de caracoles en nuestra dieta ni qué carencias orgánicas equilibra, posiblemente tendremos que consultar a un bromatólogo si las proteínas que nos proporcionan son imprescindibles para nuestra salud o para paliar alguna terrible enfermedad como las que evitan el consumo de rabos de pasas o de carne roja. Puede que sólo sean una fuente proteica más cuando el hambre aprieta o la oportunidad se nos presenta, pero lo que se presta a reflexión es la cuestión que hemos mencionado antes: ¿por qué se consumen cuando se puede obtener fácilmente carne? Desde luego no creemos que su aspecto sea más atractivo que el de un filete, por muy sanguinario que sea sacrificar vacas, cerdos o corderos, que lo es. Tampoco es probable que la ley de economía, es decir, la facilidad de obtener este recurso sea el origen de mantener esta costumbre, porque en la actualidad ambos productos se obtienen en el mismo lugar -las plazas de abastos- y con las mismas técnicas de obtención -pagando-y de preparación. Es decir, que los criterios que venimos usando para entender las preferencias en las especies de consumo del hombre a lo largo de su historia no son aplicables al caso de los caracoles. Y además, hay algo más en contra del consumo de caracoles y es que hemos de someternos al ciclo biológico del gasterópodo a pesar de que la última parte de la historia económica del hombre se basa en controlar los ciclos biológicos de animales y plantas mediante la domesticación o la preservación de los fenómenos medioambientales.

Tan sólo hallamos un argumento a favor del consumo de caracoles: el bajo coste energético que supone para el recolector directo. Pero hasta el único argumento encontrado falla en los últimos años, ya que las especies tradicionalmente recolectadas están en declive y alguna en extinción hasta su total protección por la administración, lo que hace imposible dicha actividad. Sin embargo, tanto es el gusto por estos animales que desde hace años se vienen instalando criaderos (Marasco y Murciano, 1982) y redes de importación desde el N. de África a Andalucía para que el consumo transcurra normalmente; son criaderos que no han cambiado el ciclo biológico de los caracoles ni su crecimiento, como hace la domesticación de mamíferos, sólo se modifica la producción, aumentándola tanto como lo requiere la demanda del año anterior.

Además, aún cuando este consumo no es exclusivo de nuestra región, en otros lugares como en Francia forma parte de las exquisiteces; en Andalucía es parte de nuestra dieta más cotidiana desde la primavera hasta el final del verano. Es decir, es un consumo temporal y quizás sea ésta la clave del gusto por su
Abundancia de malacofauna.

Yaciminetos de Lebrija y La Puebla de Río (Sevilla)

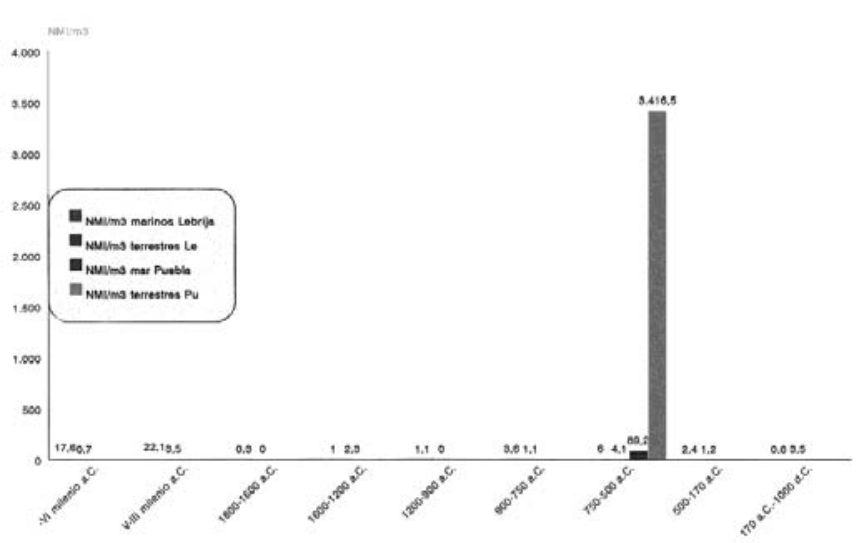

consumo: recordar nuestro origen recolector. Por otra parte, el hecho es que ésta es la realidad y tan sólo nos queda estudiar algunos aspectos históricos y paleobiológicos de esta costumbre alimenticia.

No es que encontrar esos miles de caracoles mencionados sea un hecho indiscutible del consumo humano, la distribución espacial de los mismos y el tamaño de la mayoría son los criterios usados para discriminar entre las dos maneras de morir: por muerte natural o por depredación -desde nuestro punto de vista es tan natural como la primera, pero es una forma de separar los depredadores de pequeña talla como son los agentes patógenos de los macrodepredadores que te cazan-; es decir, los muertos de los sacrificados. El tamaño adulto y óptimo de la mayoría de los ejemplares parece un inequívoco criterio para perfilar al agente recolector y consumidor que selecciona las especies y la talla de los caracoles en favor de un inmejorable balance energético: recojo lo más grande y en mayor cantidad siempre que me proporcione mejores rendimientos, es decir, con el menor gasto energético posible en relación con la energía que proporcionará lo recolectado.

Desde este punto de vista se explica que el hombre haya cambiado en algunos momentos de su historia su dieta a medida que alcanzaba los avances técnicos que hoy conocemos y su posible comienzo ecléctico, en el que no desechaba nada ya fuese recolectando con agresividad-depredación-o sin ella -recolección-, trocase en selección y crianza de algunas de aquellas especies que mejor respondieron a mantener y hasta superar el valor positivo de ese balance energético que relaciona la energía expuesta y la obtenida.

Bajo los criterios mencionados es fácil discriminar el origen de los depósitos y su relación con el hombre, la cuestión es que esta lógica puede que no responda a la realidad; y que conste que aún no hemos finalizado el estudio bioestratinómico que actualmente hacemos sobre esta relación entre la lógica y la realidad que nos plantea la presencia de un conchero. Acu-
I. Densidad de caracoles encontrados en el yacimiento arqueológico del Cerro de la Albina -La Puebla del Río- en referencia al yacimiento de Lebrija. 
$\longleftarrow$
$\square$
$\square$
Helix aspersa en el siglo VII a. C.

La Puebla de Río (Sevilla)

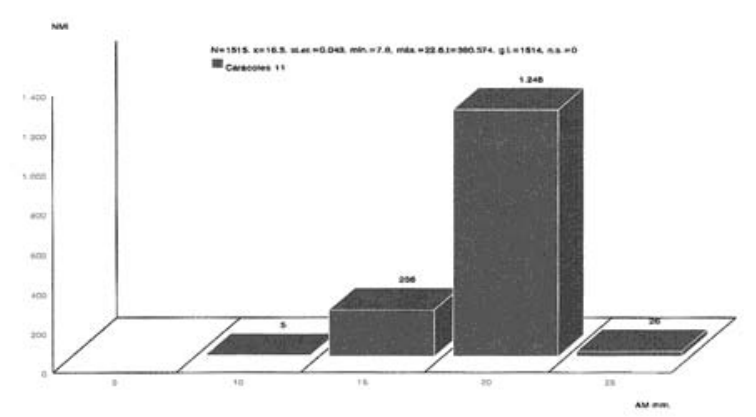

Helix aspersa en el siglo VII a. C.

La Puebla de Río (Sevilla)

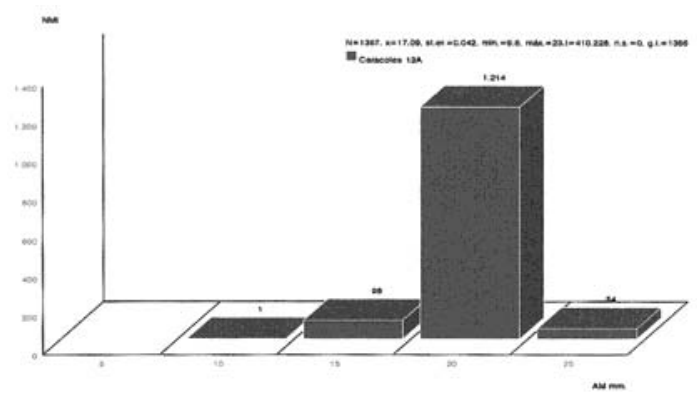

Helix aspersa en el siglo VII a. C.

La Puebla de Río (Sevilla)

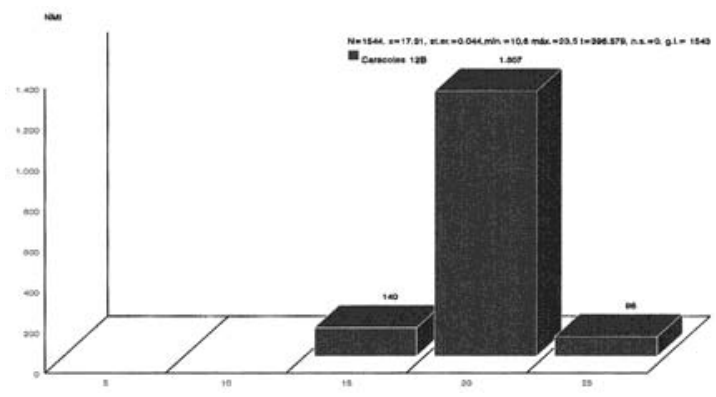

Helix aspersa en el siglo VII a. C.

La Puebla de Río (Sevilla)

2, 3, 4 y 5. Distribución en clases de tamaño de los caracoles Helix aspersa encontrados en los niveles II, 12a, 12b y cortel del fondo de cabaña del siglo VII a.C. en La Puebla del Río -Sevilla-. diendo simplemente a nuestra experiencia, el estudio bioestratinómico que venimos realizando sobre la conservación de las conchas de los caracoles terrestres nos muestra que con el tiempo las que quedan expuestas son en su mayoría de individuos adultos, dependiendo estas tanatocenosis de la especie vegetal sembrada en el parterre. E.g.: no hallamos nunca caracoles en el parterre de las salicornias, unas plantas xerófitas propias de nuestras marismas, sin embargo, en los parterres donde están sembrados los romeros hallamos caracoles y cabrillas de las especies de usual consumo actual en cantidades muy inferiores para estas últimas. Al cabo de cierto tiempo, sobre todo, de una temporada a otra la mayoría de las conchas observadas corresponden a los ejemplares de mayor tamaño. De manera que hay una criba temporal para los concheros que permanecen en la superficie que podría poner en duda el criterio del tamaño y su abundancia para discriminar la recolección humana de la muerte natural de esos caracoles.

\section{Los 6000 caracoles de La Puebla del Río}

Ha sido extraordinario el número de caracoles conservados en unas bolsadas distribuidas en cada uno de los niveles de un fondo de cabaña datado en el siglo VII-VI a.C. situado en La Puebla del Río (Sevilla) - gráfica I-. La función de estas pequeñas estructuras en el relleno es un tema de estudio del arqueólogo -Dr. J. L. Escacena- quién las relaciona con la actividad metalúrgica, pero nosotros trataremos este hallazgo desde el punto de vista tafonómico y biológico para deducir el posible origen de estos depósitos y las costumbres tróficas del agente productor. En un primer estudio definiremos las características tafonómicas de estas bolsadas cuantificando la capacidad y determinando las especies, en segundo lugar describiremos el tamaño de los ejemplares de las tres especies de caracoles terrestres que se encuentran en todas las bolsadas y, por último, trataremos de demostrar la intención del agente recolector y enterrador definiendo las características de la recolección actual e infiriendo los resultados a nuestro hallazgo. Sobre todo, nos hemos centrado en el estudio morfobiométrico de la especie que compone más del 90 \% del total de los individuos, Helix aspersa, basándonos en las características biológicas de dos muestras actuales de caracoles de esta misma especie y cuyo estudio comparativo nos ha permitido deducir la evolución temporal de la recolección de caracoles en esta parte de la región y las diferencias y similitudes de tamaño entre los ejemplares recolectados en el siglo VII a.C. y la comunidad actual de consumo.

El total de ejemplares estudiados es de 625I, de los que 576 I son de la especie Helix aspersa, 269 son cabrillas -Otala lactea- y 221 caracoles cónicos de jardín -Rumina decollata-, así que el $92.2 \%$ corresponde a la primera especie y el $4.3 \%$ y el $3.5 \%$ a las otras dos especies, respectivamente. De ese total, 1413 ejemplares pertenecen al corte I del fondo de cabaña excavado, $22.6 \%$ del total estudiado, que muestra esta frecuencia de ejemplares: 


\begin{tabular}{lrr} 
Helix aspersa & 1336 & $94.6 \%$ \\
\hline Otala lactea & 76 & $5.3 \%$ \\
\hline Rumina decollata & 1 & $0.1 \%$ \\
\hline
\end{tabular}

Los 4838 restantes se encontraron en un segundo corte repartidos en los niveles II y 12 que componen la secuencia excavada del fondo de cabaña:

\begin{tabular}{lrr} 
Helix aspersa & 4425 & $91.5 \%$ \\
\hline Otala lactea & 193 & $4 \%$ \\
\hline Rumina decollata & 220 & $4.5 \%$. \\
\hline
\end{tabular}

Quizás estas cantidades totales no digan mucho, pero siguiendo el estudio comparativo que venimos haciendo con el material óseo podemos ver en la gráfica I la densidad de caracoles respecto a la hallada en Lebrija en cualquier nivel de los estudiados y, también, observar que esta alta densidad relativa es debido a los caracoles terrestres. Es obvio también que la representación de cada especie es similar en ambas campañas y veremos que esto mismo ocurre en cada nivel del fondo de cabaña. Es decir, quienes formaron estos pequeños depósitos repitieron el esquema con gran exactitud en cuanto a la capacidad del agujero y las especies enterradas.

\section{Los niveles del fondo de cabaña}

Ya hemos mencionado los dos niveles en los que se diferenció el fondo y veremos si esta división responde a alguna diferenciación de tipo tafonómico o biológico determinando las características de cada nivel y subnivel. El hecho de carecer del volumen de los subniveles 12a, 12b y 12c nos obliga a estimar la densidad de caracoles de los dos niveles que hallamos muy próximas, 3000 individuos $/ \mathrm{m}^{3}$ para el nivel superior II y 3097 individuos/m3 para el nivel I2, una homogeneidad que sorprenderá en casi todos los datos numéricos que trataremos a lo largo del estudio.

Del nivel I I del fondo de cabaña se contabilizaron 1710 individuos, el $27.4 \%$ del total de los caracoles estudiados y el $35.3 \%$ del total del segundo corte, repartidos entre:

\begin{tabular}{lrr} 
Helix aspersa & 1515 & $88.6 \%$ \\
\hline Otala lactea & 55 & $3.2 \%$ \\
\hline Rumina decollata & 140 & $8.2 \%$ \\
\hline
\end{tabular}

En el nivel inferior 12 a tenemos 1449 caracoles, el $23.2 \%$ de los caracoles estudiados y el $30 \%$ del segundo corte, con esta distribución:

\begin{tabular}{lrr} 
Helix aspersa & 1367 & $94.3 \%$ \\
\hline Otala lactea & 54 & $3.7 \%$ \\
\hline Rumina decollata & 28 & $2.9 \%$ \\
\hline
\end{tabular}

Y en el último nivel del fondo de cabaña con restos malacológicos 12b encontramos 1679 ejemplares, el $26.9 \%$ del total estudiado y el $34.7 \%$ de la secuencia del fondo de cabaña con las mismas especies representadas en estas proporciones:

\begin{tabular}{lrr} 
Helix aspersa & 1543 & $91.9 \%$ \\
\hline Otala lactea & 84 & $5 \%$ \\
\hline Rumina decollata & 52 & $3.1 \%$ \\
\hline
\end{tabular}

Según estos resultados las bolsadas tienen una determinada capacidad que ronda los 1500 ejemplares de sólo tres especies de caracoles terrestres entre los que predominan los Helix aspersa en proporciones muy similares en los tres niveles, sobre el $90 \%$, y reseñamos la ausencia de malacofauna en el último nivel de la cabaña. No sabemos si esta determinada representación de las especies son una muestra de la antigua comunidad malacológica, sólo sabemos que esa especie suele ser la de mayor consumo en nuestra región, al contrario de lo que ocurre con otros pueblos donde se consumen más las cabrillas; de modo que a falta de otro criterio más científico ignoramos si estamos ante una muestra de la preferencia en el consumo o de la abundancia de la población natural. En estos días realizamos un muestreo malacológico en unos parterres sembrados con diferentes especies vegetales y hallamos que es mucho mayor la frecuencia de caracoles Helix aspersa y otras especies similares en talla que de cabrillas -Otala lactea- o de ruminas -Rumina decollata-, las proporciones y su representatividad en la comunidad serán expuestas en trabajos posteriores.

Tanta homogeneidad en el tamaño de las bolsadas, en la densidad de ejemplares en cada nivel y en las especies presentes sería lógico interpretarla como un indicador de algunas costumbres tróficas de aquellos agentes que produjeron su enterramiento. Primero, por las características expuestas, segundo porque no es lógico que estas cáscaras fueran arrojadas a un agujero, como el desecho de un guiso de caracoles debido a razones higiénicas, porque no generan olor alguno. Y por último, a simple vista es obvia la recolección selectiva de los ejemplares enterrados de dos de las especies que nos lleva a entender un doble origen del depósito. Para demostrar esto hemos realizado un estudio morfobiométrico que conlleva dos objetivos: demostrar que la recolección es la principal actividad de origen de las bolsadas y conocer las diferencias morfobiométricas con la población actual.

\section{Caracoles para el consumo}

Si las bolsadas de caracoles son productos de la recolección selectiva es lógico que sea una actividad rentable en la que se considere que es más productivo recoger los elementos más grandes, siempre que el balance energético sea positivo, es decir, que no cueste más energía recoger un ejemplar que la que nos va a proporcionar. De modo, que necesitamos conocer el rango de tamaño que se suele seleccionar para el consumo de estos invertebrados. Como la especie predominante es Helix aspersa nos limitamos al estudio de dos muestras de esta especie con 78 y 279 ejemplares, respectivamente, producto del tapeo de unos contertulios en un bar de Sevilla en los meses de mayo y junio de 1998. La razón de recolectar en este lugar, y no en el campo, es que se que- 


$\longleftarrow$
$\square$

ría conocer los parámetros en los que se mueve el consumo humano actual y evitar una actividad prohibida por la ley. Con la medición del diámetro máximo (AM), la variable más apreciada por nuestro ojo cuando recolectamos, obtuvimos un criterio para determinar cuál es el tamaño mínimo de consumo.

Observamos que la asociación de caracoles consumidos en mayo tenía un mínimo de $12.3 \mathrm{~mm}$ y un máximo de $20.1 \mathrm{~mm}$ y para la de junio estos valores eran de $11.1 \mathrm{~mm}$ y $20.7 \mathrm{~mm}$, respectivamente. De esta manera todos los caracoles con más de II mm de diámetro máximo pueden ser producto de la recolección y del consumo siempre que la frecuencia de ejemplares con estas medidas sea alta.

Para conocer si la mayor parte de ellos tenían estas medidas, hecho que nos confirmaría su origen, los dividimos en clases de tamaño. Los resultados nos indican que la totalidad de los caracoles de la especie Helix aspersa encontrados en todas las bolsadas tie-

\begin{tabular}{|c|c|c|c|c|c|c|c|c|}
\hline $\begin{array}{l}\text { Helix } \\
\text { aspersa }\end{array}$ & $\begin{array}{c}N-11 \\
N=1515\end{array}$ & $\mathrm{~N}-11 \%$ & $\begin{array}{c}N-12 a \\
N=1367\end{array}$ & $\mathrm{~N}-12 \mathrm{a} \%$ & $\begin{array}{l}N-12 b \\
N=1543\end{array}$ & $\mathrm{~N}-12 \mathrm{~b} \%$ & $\begin{array}{c}C-1 \\
N=1336\end{array}$ & C- $-1 \%$ \\
\hline $5-10 \mathrm{~mm}$ & 5 & 0.3 & & 0.07 & 0 & 0 & 0 & 0 \\
\hline $10-15 \mathrm{~mm}$ & 236 & 15.6 & 98 & 7.2 & 140 & 9.1 & 104 & 7.8 \\
\hline $15-20 \mathrm{~mm}$ & 1248 & 82.4 & 1214 & 88.8 & 1307 & 84.7 & 1182 & 88.5 \\
\hline $20-25 \mathrm{~mm}$ & 26 & 1.7 & 54 & 4 & 96 & 6.2 & 50 & 3.7 \\
\hline
\end{tabular}

Tabla I.

Representamos el diámetro máximo en las cuatro clases de tamaño que hemos encontrado tanto en el nivel II como en los subniveles I2a, I 2b y I2c y en el corte I realizado en este mismo fondo de cabaña del sigglo VII a.C. del yacimiento situado en el Cerro de la Albina de La Puebla del Río (Sevilla). $\mathrm{N}=$ número de ejemplares y frecuencia \%.

\begin{tabular}{|lrrrrrrrr|}
\hline $\begin{array}{l}\text { Otala } \\
\text { lactea }\end{array}$ & $\begin{array}{c}\mathrm{N}-11 \\
\mathrm{~N}=55\end{array}$ & $\mathrm{~N}-11 \%$ & $\begin{array}{c}\mathrm{N}-12 \mathrm{a} \\
\mathrm{N}=54\end{array}$ & $\mathrm{~N}-12 \%$ & $\begin{array}{c}\mathrm{N}-12 \mathrm{~b} \\
\mathrm{~N}=84\end{array}$ & $\mathrm{~N}-12 \mathrm{~b} \%$ & $\begin{array}{c}\mathrm{C}-1 \\
\mathrm{~N}=76\end{array}$ & $\mathrm{C}-1 \%$ \\
\hline \hline $15-20$ & 15 & 27.3 & 1 & 1.9 & 0 & 0 & 0 & 0 \\
\hline $20-25$ & 38 & 69.1 & 19 & 35.1 & 140 & 9.1 & 31 & 40.8 \\
\hline $25-30$ & 2 & 3.6 & 33 & 61.1 & 1307 & 84.7 & 43 & 56.6 \\
\hline $30-35$ & 0 & 0 & 1 & 1.9 & 96 & 6.2 & 2 & 2.6 \\
\hline
\end{tabular}

Tabla 2.

Es evidente que en el nivel II las cabrillas se reparten más entre las clases de tamaño más pequeñas que en el nivel 12 del fondo de cabaña y del corte I del yacimiento del Cerro de la Albina de La Puebla del Río (Sevilla).

\begin{tabular}{|lrrrrrr|}
\hline $\begin{array}{l}\text { Rumina } \\
\text { decollata }\end{array}$ & $\begin{array}{c}\mathrm{N}-11 \\
\mathrm{~N}=140\end{array}$ & $\mathrm{~N}-11 \%$ & $\begin{array}{c}\mathrm{N}-12 \mathrm{a} \\
\mathrm{N}=28\end{array}$ & $\mathrm{~N}-12 \mathrm{a} \%$ & $\begin{array}{c}\mathrm{N}-12 \mathrm{~b} \\
\mathrm{~N}=52\end{array}$ & $\mathrm{~N}-12 \mathrm{~b} \%$ \\
\hline \hline $0-2$ & 57 & 40.7 & 22 & 78.6 & 0 & 0 \\
\hline $2-4$ & 34 & 24.3 & 3 & 10.7 & 21 & 40.4 \\
\hline $4-6$ & 4 & 2.9 & 0 & 0 & 25 & 48.1 \\
\hline $6-8$ & 2 & 1.4 & 0 & 0 & 1 & 1.9 \\
\hline $8-10$ & 28 & 20 & 3 & 10.7 & 5 & 9.6 \\
\hline $10-12$ & 15 & 10.7 & 0 & 0 & 0 & 0 \\
\hline
\end{tabular}

Tabla 3.

Valores de la anchura máxima de las ruminas encontradas en los niveles del fondo de cabaña del siglo VII a.C. excavado en el Cerro de la Albina de La Puebla del Río (Sevilla). nen un tamaño dentro de los rangos mostrados por las muestras actuales de consumo que ratifica la recolección selectiva a partir de un cierto tamaño.

Tenemos en el nivel más superficial, II, que más del $98 \%$ de los caracoles Helix aspersa tienen un tamaño superior a $10 \mathrm{~mm}$ y de ellos más del $80 \%$ tienen una talla superior a los $15 \mathrm{~mm}$, resultados fácilmente observables en la gráfica 2. Esta tendencia se repite en los otros dos niveles, $12 \mathrm{a}$ y $12 \mathrm{~b}$, y en los ejemplares del corte I, como podemos comprobar en las gráficas 3, 4 y 5; con una salvedad: los caracoles de los niveles inferiores parecen más grandes que los del nivel superior, el más moderno. Un hecho que se deduce de los datos de la tabla I donde la frecuencia de la clase 10 - $15 \mathrm{~mm}$ se reduce en favor de la siguiente clase, una diferencia estadísticamente significativa como veremos en el apartado de las pautas de la recolección de la se deducirá algunas características de la temporalidad de esta actividad trófica.

\section{La recolección de cabrillas}

Para el estudio biométrico de las cabrillas -Otala lactea- no contamos con una muestra actual producto del consumo, pero acudimos a un cierto razonamiento trófico, si a partir de los 1 I mm de diámetro el recolector le parece rentable la recogida de invertebrados es lógico pensar que el tamaño mínimo de consumo de las cabrillas podría partir de aquí. Obsérvese que en las clases de talla de la tabla 2 tenemos que más del $90 \%$ de los ejemplares pertenece a los tamaños superiores a los $20 \mathrm{~mm}$, un tamaño óptimo en la recolección de caracoles. La única diferencia que advertimos está en el tamaño medio de las cabrillas del nivel I I respecto al resto de las muestras, donde queda representada la clase inferior de $15 \mathrm{~mm}$ con un $27.3 \%$ del total, observándose para este caso que el tamaño se ha desplazado a un rango inferior, una clase que no está representada en el nivel 12 y que podría indicarnos algún tipo de diferencia significativa debido a las costumbres del recolector o a las condiciones ambientales. Esta representación de las clases de talla es bastante expresiva en las gráficas 6, 7, 8 y 9 donde es evidente la ausencia de las clases más pequeñas.

\section{La inexplicable presencia de las ruminas}

Por último, la asociación de ruminas presenta otra distribución en tamaños de los ejemplares conservados, la mayor parte de los ejemplares son tan pequeños que es improbable que su recolección fuese rentable, la única referencia acerca de su tamaño es la observación realizada en los parterres de La Cartuja. Debido a su forma cónica medimos la anchura máxima de la concha observando que la distribución de esos ejemplares por clase de tamaño es más parecida a la distribución de una tanatocenosis natural donde la mortandad siempre es mayor entre las clases más jóvenes o más pequeñas, algo que podemos observar en los histogramas 10, II y 12. En la tabla 3 
observamos que apenas hay ejemplares que sobrepasen el $\mathrm{cm}$ de anchura máxima y que la mayor parte de ellos en los niveles II y 12 a, el $65 \%$ y $89.3 \%$, se da entre 0 y $4 \mathrm{~mm}$ de anchura y en el $12 \mathrm{~b}$, el $88.5 \%$, entre las clases 2 y $6 \mathrm{~mm}$. A partir de los 6 $\mathrm{mm}$ los porcentajes de ruminas son inferiores al $30 \%$ en el nivel II y al $10 \%$ en el nivel 12. De cualquier manera, no observamos la misma tendencia definida en los caracoles y en las cabrillas donde las clases más grandes y, por lo tanto adultas, son las que componen la totalidad de la asociación; mientras que en las ruminas son los ejemplares más pequeños los que mejor están representados en la muestra. Es en cierta manera otra forma de selección, pero esta vez en contra de lo que venimos reconociendo como actividad de consumo rentable, puesto que su tamaño reporta dificultad para la recogida, baja aportación alimenticia y, en consecuencia, bajo rendimiento de la actividad.

El origen de esta especie en estas bolsadas podría tener una respuesta deductiva basada en la distribución de las clases de tamaño y su similitud con las tanatocenosis naturales, de esta forma entenderíamos que su presencia es ajena a la intención del hombre y que una colonización de las ruminas del lugar donde se enterraron caracoles y cabrillas podría explicar su presencia. Por otra parte, aprovechamos el tamaño de estas ruminas para asegurarnos de que la presencia de caracoles y cabrillas adultas no es un efecto sesgado de la recogida de material durante los trabajos de excavación.

\section{Los recolectores de caracoles}

Podríamos resumir este estudio diciendo que tenemos tres asociaciones de caracoles terrestres encerradas en unos agujeros que nos indican la intencionalidad del agente enterrador apoyada en el tamaño de las bolsadas, en las especies seleccionadas y en el tamaño de los ejemplares de dos de esas especies, lo que ratifica una recolección selectiva dirigida a obtener la mayor biomasa posible. Y una tercera especie cuya tanatocenosis es la que correspondería a la mortandad de una población natural representada por las ruminas cuyo consumo no sería rentable debido al tamaño de los ejemplares. Su presencia es posible que sea debida a los restos de una población establecida en este mismo lugar, favorecida por la materia orgánica restante de los caracoles enterrados. En cualquier caso y siguiendo el criterio de que la recolección de caracoles se da a partir de un tamaño adulto, las ruminas no han sido recolectadas para el consumo.

Además, nos encontramos que aún entre los caracoles y cabrillas adultos hay diferencias de talla hasta del doble y comprobamos si esas diferencias eran significativas a la hora de entender la eficacia del recolector. El test estadístico t-Student aplicado considera que existen diferencias significativas en las tres especies, de modo que no discrimina la actividad que originó la presencia de caracoles y cabrillas de la que
Otala lactea en el siglo VII a. C.

La Puebla de Río (Sevilla)

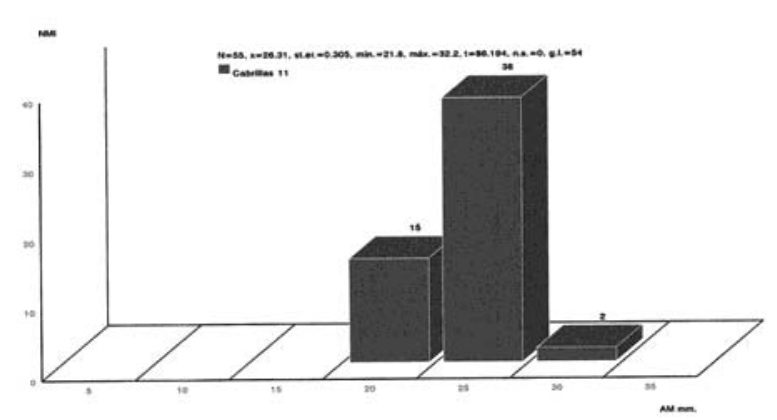

Otala lactea en el siglo VII a. C.

La Puebla de Río (Sevilla)

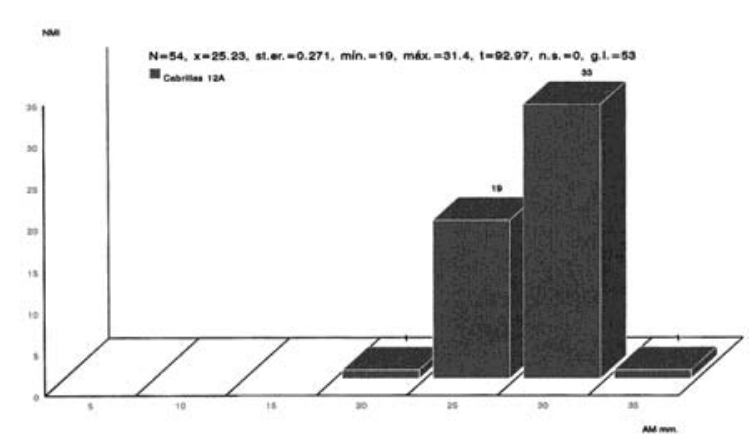

Otala lactea en el siglo VII a. C.

La Puebla de Río (Sevilla)

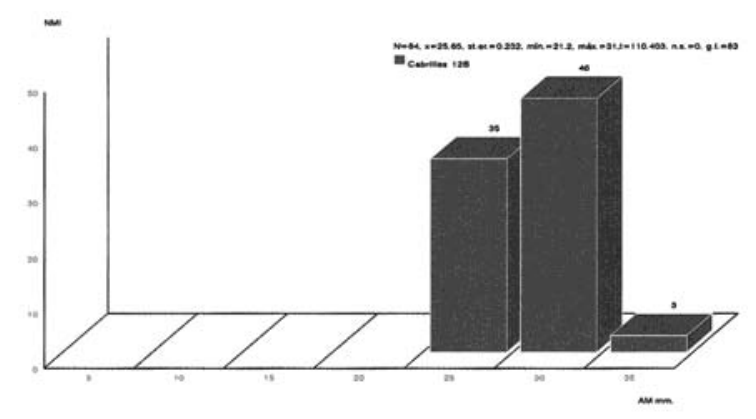

Otala lactea en el siglo VII a. C.

La Puebla de Río (Sevilla)

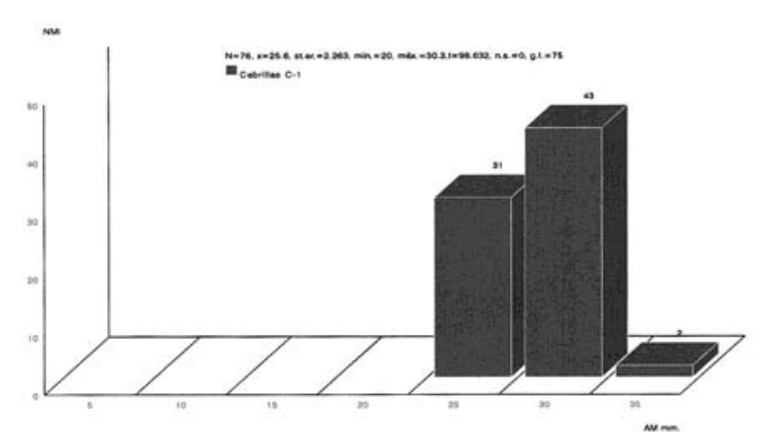

6, 7, 8 y 9. Abundancia de cabrillas Otala lactea según las clases de tamaño, conservadas en las bolsadas de los niveles del fondo de cabaña del siglo VII a.C. en La Puebla del Río -Sevilla- 


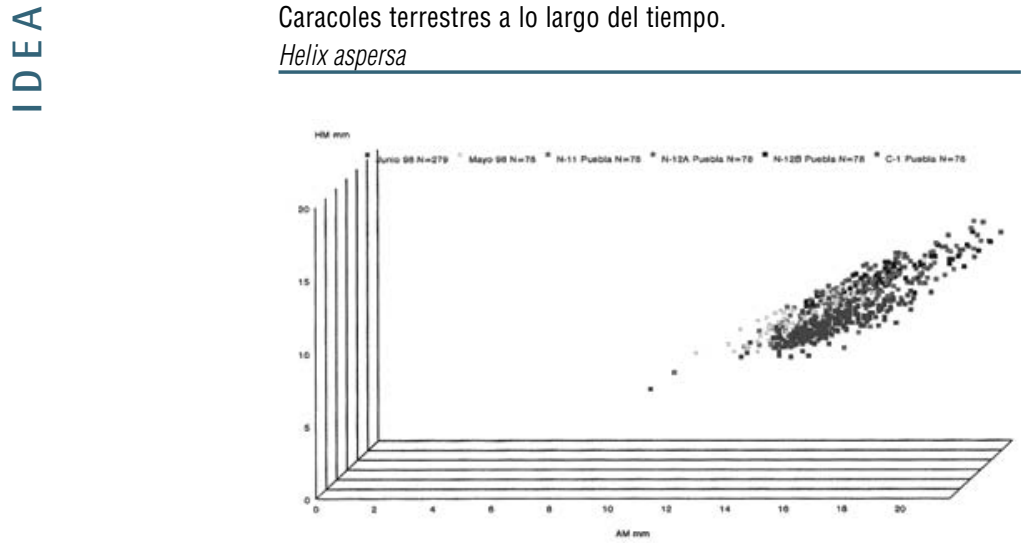

Rumina decollata en el siglo VII a. C. La Puebla del Río (Sevilla)

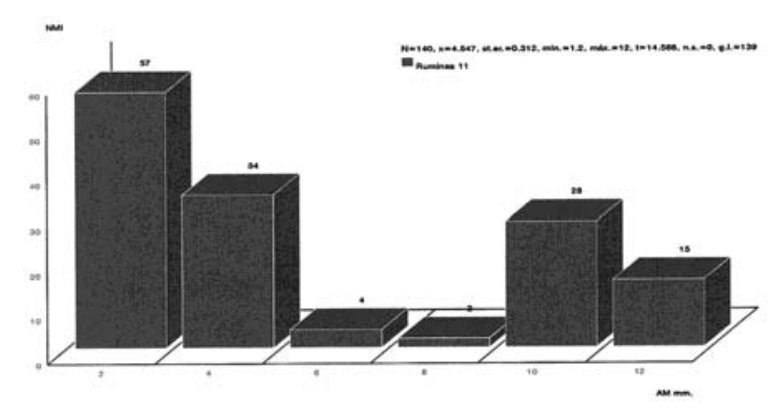

Rumina decollata en el siglo VII a. C. La Puebla del Río (Sevilla)

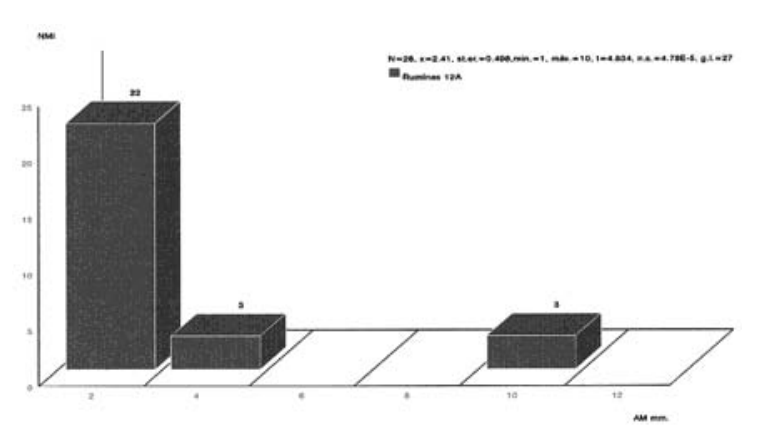

10, 11 y 12. Distribución de las ruminas en las distintas clases de tamaño. Yacimiento arqueológico del Cerro de la Albina situado en La Puebla del Río

-Sevilla-. Fondo de cabaña del siglo VII a.C. de la experiencia de quién selecciona el tamaño o del estado de la población en la época de recolección en la que ese recolector encuentra que la mayoría de los caracoles han alcanzado un tamaño óptimo. Esto último nos indicaría que se espera la época más rentable.

Centrándonos en la especie más abundante, Helix aspersa, intentamos saber si esta homogeneidad que venimos resaltando en cuanto a la capacidad de las bolsadas, a las proporciones de representación de las tres especies y al tamaño de los ejemplares es significativa, es decir, si todos los caracoles han sido recolectados en un mismo momento de su crecimiento o podemos detectar algún tipo de diferencia. Disponemos de las dos poblaciones de Helix aspersa para obtener unos criterios de crecimiento actual y extrapolarlos a las muestras antiguas.

De los ejemplares actuales medimos el diámetro máximo (AM) y la altura máxima (HM) que nos ayudaría a cuantificar la relación anchura/altura de la concha o lo que es igual el crecimiento en los meses de mayo y junio. Los valores estadísticos descriptivos de ambas muestras nos dan una media de AM para mayo de $16.13 \mathrm{~mm}$ y de $17.16 \mathrm{~mm}$ para junio, mientras que los respectivos valores de altura son más próximos, I I.28 para mayo y I I.22 para junio, el test de correlación de Pearson nos muestra una tendencia definida por esta ecuación para mayo:

$\log H M=1.150+0.67 \log A M$

$F=246.86, p=0, c c=0.87, g . l .=77$

y otra para junio:

$\log H M=1.221+0.67 \log A M$

$F=642.53, p=0, c c=0.84, g . l .=278$

que nos da idea de que ambas siguen unas tendencias similares en el crecimiento, aunque con diferencias significativas en cuanto al diámetro máximo de los caracoles de mayo y los de junio. Esta variable AM representa el crecimiento del borde de la concha que es más rápido en la juventud que en la vejez, de modo que la concha se agranda y se amplía en el borde y se refuerza progresivamente hacia el interior (Lindner, 1983), este refuerzo se mide por la altura de las conchas que, a su vez, es una variable más sometida a las condiciones ambientales. De tales resultados se deduce que estas dos asociaciones de caracoles procederían de una comarca, zona o lugar con condiciones ambientales similares y que la diferencia de diámetro máximo marca la diferencia de época de recolección, en este caso entre mayo y junio.

Con el objetivo de conocer las tendencias en el crecimiento de los caracoles del s. VII a.C. medimos esas dos variables del crecimiento (AM y HM) en 78 ejemplares elegidos al azar de cada una de las muestras subfósiles del yacimiento, asegurándonos antes de que son muestras representativas de las bolsadas y no una muestra de nuestro método de selección. Al igual que la totalidad estudiada, estos 78 caracoles 
de cada bolsada muestran diferencias significativas en sus dimensiones -valores de t-Student en anexo- . Representamos las tendencias mostradas por estos ejemplares en nubes de puntos acompañadas de sus respectivas ecuaciones que cuantifica cómo se relacionan anchura y altura de la concha -gráfica I3- como ya hicimos con los caracoles actuales utilizando el test de correlación de Pearson:

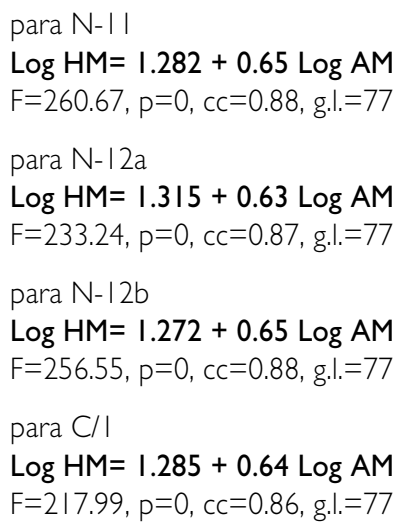

Y comprobamos si estos niveles muestran diferencias significativas en algunas de estas dos variables, el resultado del test t-Student es que el nivel I I es significativamente distinto en anchura y altura de la concha a las otras tres muestras subfósiles, es decir, son ejemplares más jóvenes y, posiblemente, criados en condiciones ambientales distintas. De ahí que la media de sus dimensiones sea algo inferior a los de los subniveles 12, entre los que no hemos encontrado diferencias significativas. Siguiendo un orden de deposición, si tenemos en cuenta que el nivel II es de posterior origen al 12 no concuerda que ambas asociaciones pertenezcan a una misma temporada de la misma población, porque el tamaño medio de los ejemplares del nivel I I debería ser mayor que la del nivel inferior. De lo que deduzco que los caracoles del nivel II fueron recolectados en otro año, puesto que si el nivel II está por encima del 12 es porque fueron recogidos con posterioridad y el estado de su crecimiento nos dice que pertenecen a un momento de la temporada más temprana que cuando se recogieron los del nivel 12. Por otra parte, tenemos los caracoles del fondo de cabaña del corte I que también muestran diferencias significativas en anchura y altura con los del nivel II, siendo éstos más pequeños que los primeros.

Aún hemos comparado este fondo de cabaña con las muestras actuales y el resultado es que el nivel II es distinto significativamente en ambas variables con las dos muestras actuales, es decir, que ya teníamos que ambas diferían en que los caracoles de mayo son menos anchos que los de junio, pues los del nivel II son aún menos anchos y menos altos -ver la talla media en el anexo-. Y también sabemos que los caracoles de la muestra actual de mayo difiere significativamente de los caracoles del fondo de cabaña - II, I2a, I 2 b y c/l-en anchura porque son más pequeños los primeros; mientras que los caracoles actuales de la muestra de junio difieren significativamente en
Rumina decollata en el siglo VII a. C.

La Puebla de Río (Sevilla)

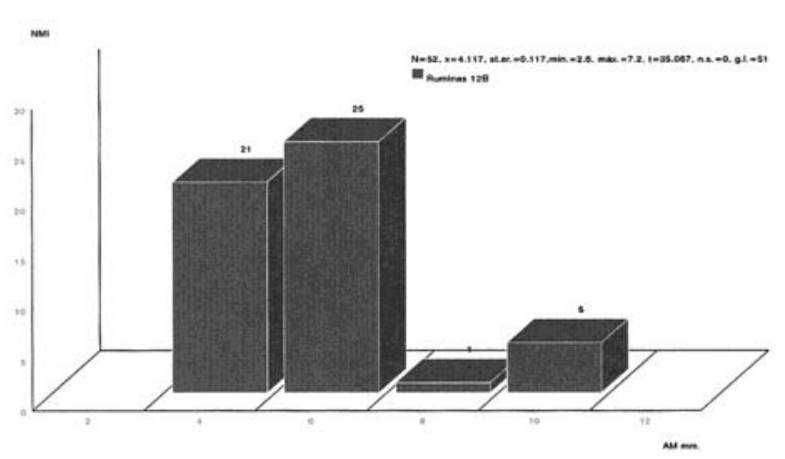

la altura respecto a todos los del fondo de cabaña, exceptuando $\mathrm{C} / \mathrm{l}$ del que difiere en anchura.

Resumiendo sabemos que los caracoles actuales de control recogidos en dos meses consecutivos de finales de la primavera del 1998 difieren significativamente en la anchura de la concha lo que nos indica que la recolección en estos dos meses se está haciendo en dos momentos distintos del crecimiento del individuo y en condiciones ambientales semejantes porque el crecimiento cíclico de la concha registra cualquier anomalía ambiental. Por otra parte, hemos aplicado este mismo método de estudio del crecimiento para ver si podemos determinar el momento de la recolección de los caracoles del fondo de cabaña y resulta que los del nivel I I difieren de los del nivel 12 que, a su vez, parecen haber sido recogidos, tanto los del 12a como los del 12b, en el mismo tiempo y de la misma población añadiéndose a éstos los del corte I. Como la única referencia sobre las diferencias de crecimiento son las muestras actuales intentamos saber cuáles son las diferencias de tamaño de éstos con los subfósiles y tenemos que los caracoles del nivel I I difieren totalmente de las muestras actuales por sus dimensiones inferiores tanto de anchura, lo que nos llevaría a pensar en una recolección más temprana a mayo, como en altura, algo que parece estar relacionado con las condiciones ambientales. Siguiendo estas premisas tenemos que el nivel 12 difiere de la muestra de mayo en anchura, son más anchos los antiguos, es decir, que los caracoles subfósiles fueron recolectados con posterioridad a mayo, y que son más altos que los de la muestra de junio, de modo que las condiciones ambientales eran distintas a las de las muestras actuales. O simplemente podríamos decir que los caracoles del nivel 12 del siglo VII a.C. recogidos en esta zona eran más grandes y los del nivel I I más pequeños que los actuales.

Aún tenemos algo más sobre estas condiciones de la recolección, los caracoles del fondo de cabaña del corte I, que hasta ahora no mostraban diferencias significativas con el nivel 12 , es decir, que serían caracoles recolectados en la misma época y con las mismas condiciones ambientales; ahora presentan diferencias significativas con la muestra de junio en
13. Mostramos las tendencias en el tamaño de las asociaciones de caracoles terrestres halladas en los niveles del fondo de una cabaña datada en el siglo VII a.C. en el Cerro de la Albina - La Puebla del Río en Sevilla- y una asociación actual procedente del consumo en los meses de mayo y junio de 1998 
cuanto a la anchura de las conchas, son algo más anchas que las actuales de junio. Esto se resumiría diciendo que hay una recolección de los caracoles primero del corte le inmediatamente después de los del nivel 12 en un momento de su crecimiento similar a los actuales de junio ocurrido en condiciones ambientales distintas a las actuales, quizás más favorables en el siglo VII a.C., que justifican su tamaño.
Así que los actuales recolectores de caracoles, al menos, los que nos proporcionaron las muestras actuales, tienen sus ancestros en La Puebla del Río, unos ancestros que ya consiguieron ser tan eficaces como los de ahora. Tan sólo encontraron condiciones ambientales algo distintas.

\section{Anexo}

Tabla de valores estadísticos

\begin{tabular}{llllllllll} 
nivel & $\mathrm{n}$ & $\overline{\mathrm{x}}$ & $\mathrm{SE}$ & mín. & máx. & rango & $\mathrm{t}$ & n.s. & g.l. \\
\hline 11 & 1515 & 16.5 & 0.043 & 7.8 & 22.6 & 14.8 & 380.57 & 0 & 1514 \\
\hline $12 \mathrm{a}$ & 1367 & 17.09 & 0.042 & 9.8 & 23 & 13.2 & 410.23 & 0 & 1366 \\
\hline $12 \mathrm{~b}$ & 1544 & 17.3 & 0.044 & 10.6 & 23.5 & 12.9 & 396.58 & 0 & 1543 \\
\hline $\mathrm{C}-1$ & 1335 & 17.02 & 0.043 & 12.1 & 22.8 & 10.7 & 399.62 & 0 & 1334 \\
\hline $\mathrm{M} 1$ & 78 & 16.13 & 0.160 & 12.3 & 20.1 & 7.8 & 99.70 & 0 & 77 \\
\hline $\mathrm{M} 2$ & 279 & 17.16 & 0.072 & 11.1 & 20.7 & 9.6 & 239.12 & 0 & 278 \\
\hline 11 & 78 & 16.64 & 0.195 & 11.2 & 21.7 & 10.5 & 85.4 & 0 & 77 \\
\hline $12 \mathrm{a}$ & 78 & 17.39 & 0.188 & 14.6 & 21.1 & 6.5 & 92.3 & 0 & 77 \\
\hline $12 \mathrm{~b}$ & 78 & 17.44 & 0.188 & 14.6 & 21.3 & 6.7 & 92.92 & 0 & 77 \\
\hline $\mathrm{C}-1$ & 78 & 17.22 & 0.185 & 14.6 & 21.9 & 7.3 & 93.08 & 0 & 77 \\
\hline
\end{tabular}

Valores estadísticos descriptivos del tamaño de la muestra - N-, de la media de talla en $\mathrm{AM}-\overline{\mathrm{X}}-$-, del error estándar -SE-, de los mínimos -mín-y máximos - máx- medidos y del rango en el que oscilan estos valores, así como de los resultados del test estadístico tStudent -t-, nivel de significación -n.s.-y de los grados de libertad -g.l.--.

\section{Referencias bibliográficas}

LINDNER, G. 1983. Moluscos y caracoles de los mares del mundo. Ed. Omega. Madrid.
MARASCO, F. \& C. MURCIANO. 1982. Guía completa de la cría de caracoles. Sistema de helicicultura de ciclo biológico completo. Ed de Vecci. Barcelona. 